\title{
Guía 2019 del Colegio Norteamericano de Cardiología y la Asociación Norteamericana del Corazón para la prevención primaria de enfermedad cardiovascular: puntos clave
}

2019 Guideline American College of Cardiology and American Heart Association for primary orevention of

cardiovascular disease: key points

\section{Comentado de:}

Arnett DK et al. Circulation. 2019 Mar 17:CIR0000000000000678. doi: 10.1161/CIR.0000000000000678. [Epub ahead of print] ${ }^{1}$

\section{Resumen del artículo original}

Gran parte de la carga de la enfermedad cardiovascular y la mortalidad se deben a cuatro hábitos no saludables (fumar, recibir una dieta deficiente, tener un elevado índice de masa corporal, llevar un estilo de vida sedentario) y tres factores de riesgo principales (hipercolesterolemia, hipertensión, diabetes). La nueva guía de prevención primaria reune y actualiza pautas de orientación sobre 9 temas: evaluación de riesgos, dieta, ejercicio/actividad física, obesidad, diabetes mellitus (DM) tipo 2, colesterolemia, hipertensión, cesación tabáquica y aspirina.

\section{0 mensajes clave de la Guía}

1. La forma más importante de prevenir la enfermedad cardiovascular (EC), la insuficiencia cardíaca y la fibrilación auricular es promover un estilo de vida saludable a lo largo de la vida.

2. El enfoque de atención en equipo es una estrategia efectiva para la prevención de EC. Los profesionales de la salud deben evaluar los determinantes sociales de la salud que afectan a las personas para informar las decisiones terapéuticas.

3. En adultos de 40 a 75 años de edad que están siendo evaluados para la prevención de EC se debe estimar el riesgo de EC 10 años y tener un discusión de riesgo entre el médico y el paciente antes de comenzar la terapia farmacológica (antihipertensivos, estatinas, o aspirina). Además, la evaluación de otros factores de riesgo podría ayudar a guiar decisiones sobre intervenciones preventivas en individuos seleccionados, como el puntaje de calcio coronario.

4. Todos los adultos deben consumir una dieta saludable que haga hincapié en la ingesta de verduras, frutas, nueces, granos, proteínas magras vegetales o animales, y pescado; y disminuir la ingesta de grasas trans, carnes procesadas, carbohidratos refinados y bebidas azucaradas. Para adultos con sobrepeso y obesidad, se recomienda el asesoramiento y la restricción calórica para lograr y mantener la pérdida de peso.

5. Los adultos deben realizar al menos 150 minutos por semana de actividad física acumulada de intensidad moderada, o 75 minutos por semana de actividad física de intensidad vigorosa.

6. Para los adultos con DM tipo 2, los cambios en el estilo de vida, como mejorar los hábitos alimenticios y el ejercicio, son cruciales. Si está indicado el tratamiento farmacológico, la metformina es la primera opción, y como segunda línea, un inhibidor de cotransportador de sodio-glucosa 2 o un agonista del receptor de péptido 1 similar al glucagón.

7. En los adultos, debe determinarse, en cada visita, el consumo de tabaco. En aquellos que fuman, debe recomendarse fuertemente la cesación tabáquica.

8. La aspirina debe usarse con poca frecuencia en la prevención primaria de rutina de EC debido a la falta de beneficio neto.

9. El uso de estatinas es un tratamiento de primera línea para la prevención primaria de EC en pacientes con niveles elevados de LDL ( $\geq 190 \mathrm{mg} / \mathrm{dL}$ ), pacientes con DM, que tienen entre 40 y 75 años de edad, y en aquellos con un riesgo suficiente de EC después de realizar una discusión de riesgo entre el médico y el paciente.

10. Las intervenciones no farmacológicas son recomendadas para todos los adultos con presión arterial elevada o hipertensión. Para aquellos que requieren terapia farmacológica, la presión arterial deseada debe ser generalmente $<130 / 80 \mathrm{mmHg}$

Conflicto de interés de los autores: Se presenta una tabla con las relaciones de los miembros del comité con la industria y otras entidades que fueron informadas por los autores.

\section{Comentario}

El Colegio Americano de Cardiología (ACC) y la Asociación Americana del Corazón (AHA) publican esta guía que reúne las recomendaciones existentes de las dos organizaciones y las combina con algunas recomendaciones nuevas. En la misma se refuerza la idea de que la mejor intervención para prevenir la EC es la promoción de un estilo de vida saludable durante toda la vida. Propone un enfoque integral centrado en el paciente, alentando un proceso de toma de decisiones compartidas (TDC) para lograr un tratamiento óptimo. Sin embargo no provee herramientas para orientar o facilitar este proceso. Sólo se incluye un checklist para orientar la discusión de riesgo con los pacientes.

En esta guía se menciona la importancia de la atención por equipos multidisciplinarios para mejorar la calidad y el mantenimiento de la prevención de EC, promoviendo la colaboración entre diferentes profesionales y la participación del paciente y los miembros de la familia para facilitar el alcance de los objetivos terapéuticos.

Un aspecto destacable es que el documento pone atención en los determinantes sociales. Sabemos que las desigualdades socioeconómicas son fuertes determinantes del riesgo de EC en todo el mundo ${ }^{2-4}$, sin embargo, en la atención individual de los pacientes, no siempre lo tenemos en cuenta y no adecuamos nuestras recomendaciones a sus características socioeconómicas, culturales, educativas, familiares y ambientales. Para mejorar esto se proponen una serie de intervenciones relacionadas con cada aspecto de la prevención primaria de enfermedad cardiovascular, por ej: riesgo cardiovascular 
(detectar factores estresantes psicosociales y proporcionar asesoramiento adecuado); alimentación (evaluar barreras para adherirse a una dieta saludable, incluyendo el acceso a los alimentos y los factores económicos); actividad física (determinar el acceso a instalaciones para la realización de actividad física y el ambiente del barrio).

Un aspecto controvertido es que las nuevas pautas fomentan el uso del puntaje de calcio coronario (CAC, por sus iniciales en inglés), al que se atribuyen propiedades de discriminación y reclasificación de riesgo bien establecidas para guiar las estrategias preventivas en adultos con riesgo intermedio o en pacientes seleccionados con riesgo límite. Sin embargo existen algunas cuestiones pendientes con respecto a su utilización: 1) No se ha establecido que instituir o intensificar la modificación del factor de riesgo farmacológico, en pacientes asintomáticos con el CAC, mejore los resultados; 2) No se ha determinado el daño potencial asociado a los falsos positivos y la dosis de radiación y 3) No se ha definido la costo-efectividad del CAC. ${ }^{5}$ Otro tema fundamental que aborda la guía es la utilización de aspirina en prevención primaria de EC. A la luz de los resultados de nuevos estudios ${ }^{6,7}$ y la evidencia acumulada en los últimos años ${ }^{8-10}$ postula que, en pacientes entre 40 y 70 años, la aspirina puede ser considerada si tienen un alto riesgo cardiovascular y un bajo riesgo de sangrado. No se recomienda la aspirina como prevención primaria en individuos $>70$ años o con riesgo aumentado de sangrado.

Entre sus limitaciones, llama la atención que, dada la amplia cobertura en la literatura reciente, la guía no revisa las recomendaciones para la evaluación de riesgos, la hipercolesterolemia y la hipertensión, siendo el punto de corte propuesto en la guía AHA sobre hipertensión arterial ${ }^{11}$ un tema sumamente controvertido ${ }^{12,13}$.

\section{Conclusiones del comentador}

Muchas de las recomendaciones por la guía resumida no son novedosas, y sólo reafirman conductas que ya se venían sugiriendo. Uno de sus aspectos más valiosos es la propuesta de un paradigma de tratamiento centrado en el paciente que hace hincapié en la atención en equipo, la toma de decisiones compartidas y la evaluación de los determinantes sociales de la salud.

\section{Carolina Carrara [ Servicio de Medicina Familiar y Comunitaria, Hospital Italiano de Buenos Aires carolina.carrara@hospitalitaliano.org.ar ]}

Carrara C. Guía 2019 del Colegio Norteamericano de Cardiología y la Asociación Norteamericana del Corazón para la prevención primaria de enfermedad cardiovascular: puntos claveEvid Actual Pract Ambul. 2019;22(1):e001997. Comentado de: Arnett DK et al. 2019 ACC/AHA Guideline on the Primary Prevention of Cardiovascular Disease. Circulation. 2019 Mar 17:CIR00000000000006678. doi: 10.1161/CIR.0000000000000678. [Epub ahead of print]

\section{Referencias}

1. Arnett DK, Blumenthal RS, Albert MA, Buroker AB, Goldberger ZD, Hahn EJ, et al. 2019 ACC/AHA Guideline on the Primary Prevention of Cardiovascular Disease [Epub ahead of print]. Circulation. 2019;Available from: 10.1161/CIR.0000000000000678.

2. Havranek EP, Mujahid MS, Barr DA, Blair IV, Cohen MS, Cruz-Flores S, et al. Social determinants of risk and outcomes for cardiovascular disease: a scientific statement from the American Heart Association. Circulation. 2015;132(9):873-98. Available from: 10.1161/CIR.0000000000000228.

3. Vilhelmsson A, Östergren P. Reducing health inequalities with interventions targeting behavioral factors among individuals with low levels of education—a rapid review. PLoS ONE. 2018;13(4):e0195774. Available from: 10.1371/journal.pone.0195774.

4. Schultz W, Kelly HM, Lisko J, Varghese T, Shen J, Sandesara P, et al. Socioeconomic status and cardiovascular outcomes: challenges and interventions. Circulation. 2018;137(20):2166-2178. Available from: 10.1161/CIRCULATIONAHA.117.029652.

5. CM GTK. Diagnostic and prognostic implications of coronary artery calcification; 2017.Available from: https://www.uptodate.com/contents/diagnosticand-prognostic-implications-of-coronary-artery-calcification.

6. ASCEND Study Collaborative Group. Effects of aspirin for primary prevention in persons with diabetes mellitus. N Engl J Med. 2018;379(16):15291539. Available from: 10.1056/NEJMoa1804988.

7. Gaziano J, Brotons C, Coppolecchia R, Cricelli C, Darius H, Gorelick PB, et al. Use of aspirin to reduce risk of initial vascular events in patients at moderate risk of cardiovascular disease (ARRIVE): a randomised, double-blind, placebo controlled trial. Lancet. 2018;392(10152):1036-1046. Available from: 10.1016/S0140-6736(18)31924-X.

8. Capodanno D, Angiolillo D. Aspirin for primary cardiovascular risk prevention and beyond in diabetes mellitus. . Circulation. $2016 ; 134(20): 1579-1594$. Available from: 10.1161/CIRCULATIONAHA.116.023164.

9. Pignone M, Alberts MJ, Colwell JA, Cushman M, Inzucchi SE, Mukherjee D, et al. Aspirin for primary prevention of cardiovascular events in people with diabetes: a position statement of the American Diabetes Association, a scientific statement of the American Heart Association, and an expert consensus document of the American College of Cardiology Foundation. Circulation. 2010;121(24):2694-2701. Available from: 10.1161/CIR. 0b013e3181e3b133.

10. Halvorsen S, Andreotti F, ten Berg JM, Cattaneo M, Coccheri S, Marchioli R, et al. Aspirin therapy in primary cardiovascular disease prevention: a position paper of the European Society of Cardiology working group on thrombosis. J Am Coll Cardiol. 2014;64(3):319-327. Available from: 10.1016/j.jacc.2014.03.049.

11. Whelton PK, Carey RM, Aronow WS, Casey DE, Collins KJ, Dennison-Himmelfarb C, et al. 2017 ACC/AHA/AAPA/ABC/ACPM/AGS/APhA/ASH/ASPC/NMA/PCNA Guideline for the Prevention, Detection, Evaluation, and Management of High Blood Pressure in Adults: A Report of the American College of Cardiology/American Heart Association Task Force on Clinical Practice Guidelines. . J Am Coll Cardiol. 2018;71(19):e127-e128. Available from: 10.1016/j.jacc.2017.11.006.

12. Sociedad Argentina de Cardiología. Toma de posición frente a las nuevas guías de prevención, detección, evaluación y manejo de la Hipertensión Arterial en adultos, publicadas recientemente en forma conjunta por la AAC/AHA/AAPA/ABC/ACPM/AGS/APhA/ASH/ASPC/NMA/PCNA 2017. 2017. Available from: http://www.sac.org.ar/institucional/toma-de-posicion-del-consejo-argentino-de-hipertension-arterial/.

13. Sociedad Argentina de Hipertension Arterial. Especialistas argentinos recomiendan actuar con cautela y atenerse a los lineamientos nacionales. A propósito del cambio del valor que define la condición de hipertenso en EE.UU; 2017. Available from: http://www.saha.org.ar/prensa/detalle/ especialistas-argentinos-recomiendan-actuar-con-cautela- $y$-atenerse-a-los-lineamientos-nacionales. 